\title{
SNP@Evolution: a hierarchical database of positive selection on the human genome
}

\author{
Feng Cheng1,2, Wei Chen ${ }^{1,2}$, Elliott Richards ${ }^{3,4}$, Libin Deng1,5 and \\ Changqing Zeng*1,5
}

Address: ${ }^{1}$ Beijing Institute of Genomics, Chinese Academy of Sciences, Beijing, PR China, ${ }^{2}$ Graduate School of the Chinese Academy of Sciences, Beijing, PR China, ${ }^{3}$ Department of Biology, College of Life Sciences, Brigham Young University, Provo, UT, USA, ${ }^{4}$ Current address: Baylor College of Medicine, Houston, TX, USA and ${ }^{5}$ Current address: Medical College of Nanchang University, Nanchang, Jiangxi, PR China

Email: Feng Cheng - chengf@big.ac.cn; Wei Chen - chenw@big.ac.cn; Elliott Richards - elliott.richards@byu.edu; Libin Deng - denglb1937@gmail.com; Changqing Zeng* - czeng@genomics.org.cn

* Corresponding author

Published: 5 September 2009

BMC Evolutionary Biology 2009, 9:22I doi:|0.1/86/I47|-2|48-9-22।
Received: 25 November 2008

Accepted: 5 September 2009

This article is available from: http://www.biomedcentral.com/I47I-2I 48/9/22I

(c) 2009 Cheng et al; licensee BioMed Central Ltd.

This is an Open Access article distributed under the terms of the Creative Commons Attribution License (http://creativecommons.org/licenses/by/2.0), which permits unrestricted use, distribution, and reproduction in any medium, provided the original work is properly cited.

\begin{abstract}
Background: Positive selection is a driving force that has shaped the modern human. Recent developments in high throughput technologies and corresponding statistics tools have made it possible to conduct whole genome surveys at a population scale, and a variety of measurements, such as heterozygosity $(\mathrm{HET}), F_{S T}$, and Tajima's $D$, have been applied to multiple datasets to identify signals of positive selection. However, great effort has been required to combine various types of data from individual sources, and incompatibility among datasets has been a common problem. SNP@Evolution, a new database which integrates multiple datasets, will greatly assist future work in this area.

Description: As part of our research scanning for evolutionary signals in HapMap Phase II and Phase III datasets, we built SNP@Evolution as a multi-aspect database focused on positive selection. Among its many features, SNP@Evolution provides computed $F_{S T}$ and HET of all HapMap SNPs, 5+ HapMap SNPs per qualified gene, and all autosome regions detected from whole genome window scanning. In an attempt to capture multiple selection signals across the genome, selection-signal enrichment strength $\left(E_{S}\right)$ values of HET, $F_{S T}$, and $P$-values of $i H S$ of most annotated genes have been calculated and integrated within one frame for users to search for outliers. Genes with significant $E_{S}$ or $P$-values (with thresholds of 0.95 and 0.05 , respectively) have been highlighted in color. Low diversity chromosome regions have been detected by sliding a $100 \mathrm{~kb}$ window in a $10 \mathrm{~kb}$ step. To allow this information to be easily disseminated, a graphical user interface (GBrowser) was constructed with the Generic Model Organism Database toolkit.
\end{abstract}

Conclusion: Available at http://bighapmap.big.ac.cn, SNP@Evolution is a hierarchical database focused on positive selection of the human genome. Based on HapMap Phase II and III data, SNP@Evolution includes 3,619,226/I,389,498 SNPs with their computed HET and $F_{S T}$, as well as qualified genes of $21,859 / 21,099$ with $E_{S}$ values of HET and $F_{S T}$. In at least one HapMap population group, window scanning for selection signals has resulted in I,606/10,138 large low HET regions. Among Phase II and III geographical groups, 660 and 464 regions show strong differentiation. 


\section{Background}

Natural selection has played an essential role in the formation of the human genome and in the diversity of phenotypes. The identification of the functional targets of positive selection, however, has been a major challenge in understanding the evolution of human beings. Traditional investigations to localize loci that have undergone selection have focused on the coding sequences of individual genes. For example, since the finding of hemoglobin $\mathrm{B}$ gene under the selective pressure for malaria resistance, only a limited number of genes, including G6PD, LCT, and ASPM, have been functionally determined as targets of positive selection [1-3]. In recent years, the rapid development in high throughput DNA technologies, as well as in statistical analysis and bioinformatics tools, has promoted whole genome surveys in multiple aspects of genetic variation [4-6]. Since the construction of the human HapMap, many massive genome-wide projects aiming to search and scan for SNPs, indels, copy number variations (CNVs), functional DNA elements, DNA methylation sites, and expression quantitative trait loci (eQTL) have been accomplished or undertaken [717].

These enormous maps and datasets have made great contributions to trace our evolutionary history. Although only a handful of genome-wide measurements with limited marker density have been developed to detect selection signals, these initial studies suggest that large scale and highly detailed analyses will greatly illuminate our understanding of human evolution [18-21]. Using whole genome SNP data, selection signals may be demonstrated through the computation of classical measurements including heterozygosity (a measurement which is used to estimate the frequency of heterozygote in a population, also referred to as HET), Tajima's D (a statistical test which is used to determine whether a genetic locus is under neutral selection), and the fixation index (a measurement which is used to compare the genetic variability within and between populations, also referred to as $F_{S T}$ ) $[18,22,23]$. Moreover, haplotype based measurements-such as extended haplotype homozygosity (EHH) and relative $\mathrm{EHH}(\mathrm{REHH})$, or more complex values including integrated haplotype homozygosity $(\mathrm{iHH})$ and integrated haplotype score (iHS)--have been successfully applied to find signals of recent positive selection across the human genome [24,25]. A few databases, such as Haplotter and SNP@Ethnos, serve as public tools in population genetics [26]. Built to find ethnically related SNPs, SNP@Ethnos provides 100,736 individual variants with large ethnic differences. With the determination of ancestral or derived allele state, Haplotter is efficient in finding recent positive selections and their affected genes by the frequency of haplotypes that extend from a core SNP.
To complement these and other publicly available databases, we have built an integrated data library on human evolution ("SNP@Evolution") which offers several novel features: (1) the inclusion of $E_{S}$ (enrichment strength of selection-signals) to estimate the selection strength on a specific gene by computation of outlier ratios; (2) a sliding window scanning method that uses measures of HET and $F_{S T}$ to locate genomic segments under selection (with the resulting regional signals reflecting geographical adaptation, founder effects, and fixed or unfixed selections); (3) HapMap Phase III data from samples of 11 populations; (4) the integration with a haplotype-based dataset of iHS that simplifies the process of comparing multiple datasets by providing a simple, easy-to-read table.

One of SNP@Evolution's primary functions is to find selection signals from both chromosome regions and individual SNPs. To achieve this aim, our major strategy has involved the computation and comparison of HET, $F_{S T}$, and iHS of all SNPs and their regional values using a sliding window method, then followed by the demonstration of outliers of each measurement as the selection signals. $E_{S}$ values of various measurements in most annotated genes are also listed as an independent dataset. Selection signals can therefore be detected in the data query or visualization interface by (1) SNP outliers, (2) low HET regions merged from adjacent window outliers, and (3) $\mathrm{E}_{\mathrm{S}}$ values of individual genes. With the comparison and integration of multiple datasets, SNP@Evolution provides candidate genes and regions that will assist researchers in locating positive selection signals genomewide.

\section{Construction and content Data source}

The strategy of our database construction and data processing route is illustrated in Fig. 1. Genotype datasets are derived from the International HapMap Phase II and Phase III data repository (release 21\# NCBI build35, release 24\# NCBI build36, release 26\# NCBI build36, http://www.hapmap.org) [7,8]. Only data of unrelated individuals were utilized. For HapMap Phase II, samples of 60 Utah residents with ancestry from northern and western Europe (CEU), 45 Han Chinese in Beijing (CHB), 45 Japanese in Tokyo (JPT), and 60 Yoruba in Ibadan, Nigeria (YRI) were included. Considering the great genetic similarity between CHB and JPT, we pooled the data of both as one geographical group and denoted it as ASN (Asian). Phase III data came from samples of 11 populations, including $84 \mathrm{CHB}, 85$ Chinese in Metropolitan Denver, Colorado (CHD), 86 JPT, 113 CEU, 88 Toscans in Italy (TSI), 113 Yoruba in Ibadan, Nigeria (YRI), 53 African ancestry in Southwest USA (ASW), 90 Luhya in Webuye, Kenya (LWK), 143 Maasai in Kinyawa, Kenya (MKK), 88 Gujarati Indians in Houston, Texas (GIH), 50 

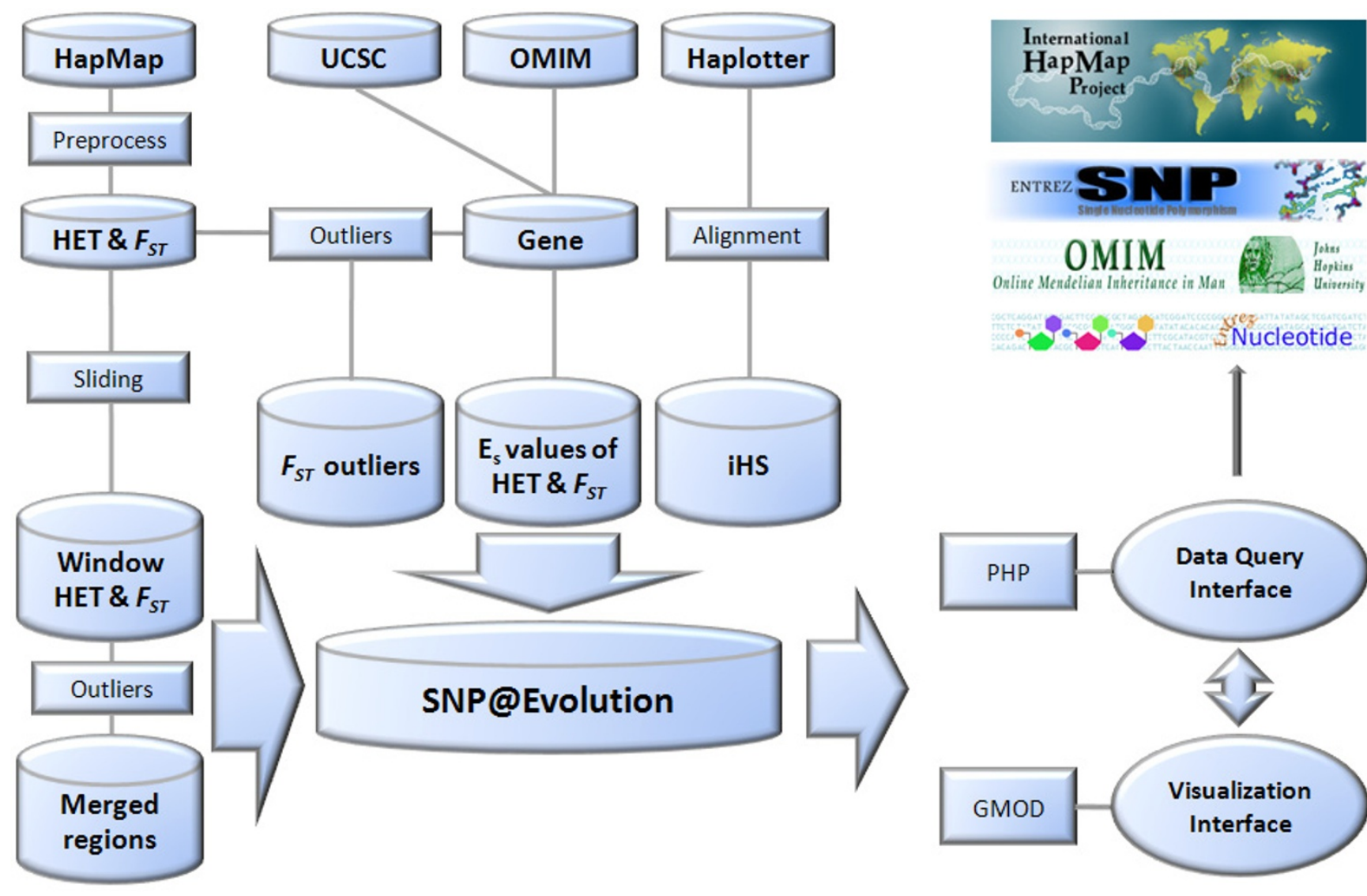

Figure I

Data collecting and processing strategy for building SNP@Evolution. HapMap, UCSC, OMIM, and Haplotter were major sources for SNP, Gene, and iHS. After computation and processing with a sliding window and empirical distribution method, we obtained five types of outcomes that may contain evolutionary signals in the human genome including low HET or highly differentiated regions, $F_{S T}$ outliers of SNPs, $E_{S}$ values of HET and $F_{S T}$ on genes, and SNP outliers of iHS. In order to visualize these datasets, we used PHP and GMOD to build the table and figure interfaces. Links to the public online databases, including Entrez Nucleotide, dbSNP, OMIM, and HapMap, are provided together with the query results in SNP@Evolution.

Mexican ancestry in Los Angeles, California (MEX). According to their continental origination, samples were then divided into four geographical groups, i.e., CHB, CHD, and JPT grouped as ASN (Eastern Asian ancestry); CEU and TSI as EUR (European ancestry); YRI, ASW, LWK, and MKK as AFR (African ancestry); and GIH and MEX as AME (Native American ancestry). The iHS dataset (from HapMap phase II), including iHS of SNPs and $P$-values on iHS from 12,683 genes, was retrieved from Haplotter [25].

\section{Data analysis}

We first computed HET and Akey's $F_{S T}$ to measure the polymorphism within each population and the differentiation among geographical groups [18]. For gene analysis, we considered a genic region to be a gene with $2 \mathrm{~kb}$ flanking regions. Genic regions containing no less than 5 genotyped SNPs in the dataset were chosen for subsequent analysis. From 24,011 annotated genes in NCBI build36, we identified 21,859 and 21,099 qualified genic regions in HapMap Phase II and Phase III, respectively.

To search for outliers of HET and $F_{S T}$, the SNPs with HET values larger than average were considered as outliers directly. For $F_{S T}$, we defined an "outlier" as a SNP whose value extends beyond the nearest quartile by a length of at least 1.5 times the inter-quartile range. With simulated data, we first sought to determine whether this method (henceforth referred to as the "empirical distribution method") is efficient and reliable to obtain selection signals without having to consider the apriori distributions. Genotype data under different selection strength and neutral selection generated by SelSim were mixed together [27], then the empirical distribution method was applied to find outliers. The results indicated that the empirical distribution method separated data under positive selection from the mixed data pool with a high sensitivity, sta- 
bility, and low error rate (manuscript in preparation). (To test the empirical distribution, the positive predictive value, or PPV, can be used to describe the proportion of outliers that have been the targets of selection [28]. For instance, when the simulated proportion of selection target is 0.05 with a certain recombination environment-Rec rate $=10^{-8}$, the PPV of the empirical distribution ranges from 0.50 to 0.90 under the selection strength 20200.) Therefore, we used the empirical distribution method to identify SNP outliers of $F_{S T}$ as well as the window outliers of HET (see below).

The ratios of outlier numbers of both HET and $F_{S T}$ in each genic region to the corresponding total SNPs were then calculated. Relevant enrichment value $\mathrm{E}_{\mathrm{S}}$ of both HET and $F_{S T}$ were determined as the percentile values on the distribution of outlier ratios for all qualified genes. Thus, the $\mathrm{E}_{\mathrm{S}}$ value of HET or $F_{S T}$ in a particular gene represents the enrichment strength of the outliers of each measure. (Distributing from 0 to 1 , a larger $E_{S}$ value indicates a higher outlier ratio of the corresponding measure in a gene.)

To estimate regional genetic diversity, we set up a $100 \mathrm{~kb}$ window and slid it with a $10 \mathrm{~kb}$ step throughout the human autosomes to obtain the averages of HET and $F_{S T}$. This window scanning resulted in 267,069 and 266,650 regions (with $90 \mathrm{~kb}$ overlapping each other) in HapMap Phase II and Phase III data, respectively. Regional HET was first normalized within each chromosome and then the empirical distribution method described above was used to obtain window outliers of $F_{S T}$ and normalized HET. Adjacent window outliers were merged to regions and the qualification of each region was determined by a bootstrap test. Briefly, we picked 1,000 regions randomly with the similar length to the testing region, and then calculated the HET values of these picked regions plus the testing region. The percentile values of these 1,001 HET values were defined as $P_{b o o t}$ and only those with $P_{b o o t}<$ 0.01 were accepted as low HET regions.

Consequently, 1,606 low HET regions were obtained from Phase II Build36 data, including 434 in ASN, 576 in CEU, and 596 in YRI. In addition, merged window outliers of $F_{S T}$ identified 660 highly differentiated regions among three groups. Moreover, 10,138 low HET regions were found from Phase III data, including 807 in CHB, 802 in CHD, 803 in JPT, 995 in CEU, 1,000 in TSI, 881 YRI, 965 in ASW, 916 in LWK, 962 in MKK, 1,023 in GIH, 984 in MEX. Additionally, 464 regions show strong differentiation among four geographical groups.

\section{Datasets containing evolutionary signals}

To help users locate selection signals, SNP@Evolution describes the population genetic variations of chromosome regions, genes, and SNPs with measurements of $F_{S T^{\prime}}$
HET and iHS (Fig. 2). The iHS obtained from Haplotter were first aligned with the corresponding HapMap SNPs. Of the 3,619,226 SNPs in HapMap Phase II Build36 that we computed with $F_{S T}$ and HET data, 2,663,137 SNPs also contain iHS information in Haplotter. Using the criteria of $|\mathrm{iHS}| \geq 2$ as defined in a previous study [25], 79,149, 86,272 , and 100,624 SNP outliers of iHS in ASN, CEU, and YRI, respectively, were then obtained. In addition, 33,601 SNP outliers of high $F_{S T}$ from the empirical distribution method were found. As shown in Table 1, signals of large population differentiation are shown with the trend of increased strength from intergenic to genic regions $\left(\chi^{2}\right.$ test, $\left.P=0.0000\right)$, suggesting that functional regions of the genome tend to be the targets of geographic selection. Additionally, the 5' UTR has the highest ratio of outliers. The outlier ratio in the 5' UTR is higher than other genic sections $\left(\chi^{2}\right.$ test, $P=0.0366,0.0433$, and 0.0728 for coding sequence, intron, and 3' UTR, respectively), implying the regulatory regions may play important roles in geographic differentiation.

As for all individual 24,011 genes listed with annotations, 21,859 genes with 5 or more genotyped SNPs in their genic regions are provided with $\mathrm{E}_{\mathrm{S}}$ values of HET and $F_{S T}$, resulting in 1,094 genes of low HET which exceed the $E_{S}$ threshold of 0.95. In these 1,094 genes, ASN and CEU share 370, ASN and YRI share 147, CEU and YRI share 169 , and all three groups share 81 . Such a pattern--nonAfricans sharing twice the number of genes containing enriched selection signals in comparison with that between non-African and African populations--suggests either geographical selection or founder effects by ancestors of ASN and CEU after their migration out of Africa. (Accordingly, we performed simulations based on both selection and neutral models. Very little founder effect was revealed (data not shown), suggesting that the selection events most likely resulted in these signals in genes. Our analysis results are consistent with recent reports which showed also geographic selections rather than founder effects as the major force of evolution in large genomic regions $[29,30]$.) For the 535 genes of high $F_{S T}$ ( $E_{S}$ threshold of 0.95$), 369$ were seen in at least one population which showing low HET (247 genes in ASN, 163 genes in CEU, and 42 in YRI respectively). In addition, $P$ values of iHS are included in 10,375 genes. For those with significant iHS $(P<0.05), 441,527$, and 433 genes are in ASN, CEU, and YRI, respectively, among which 32/441, $43 / 527$, and $41 / 433$ are also shown as low HET.

There are 1,389,498 genotyped SNPs in HapMap Phase III Build36, and among them, 351 outliers of $F_{S T}$ were found. $\mathrm{E}_{\mathrm{S}}$ values of HET and $F_{S T}$ in 21,099 qualified genic regions of all 11 populations are provided in our database. Finally, in our study we have found that for a fixed sample size and in regions with the same number of polymorphic 
Search Results of Gene: edar

1 regions with strong selection signals:
\begin{tabular}{|c|c|c|c|c|}
\hline Population & Chr & Start & Stop & HET_average \\
\hline ASN & 2 & 108930000 & 109100000 & 0.0536 \\
\hline
\end{tabular}

Gene statistics

\begin{tabular}{|c|c|c|c|c|c|c|c|c|c|c|c|c|}
\hline Seq_ID & Symbol & OMIM & Chr & Start & Stop & $E_{s}$ HET (A) & $E_{s,}$ HET (C) & $E_{s,}$ HET $(Y)$ & $P_{-} \mathrm{iHS}(\mathrm{A})$ & $P_{-}$iHS (C) & $P_{-}$iHS $(Y)$ & $\mathrm{E}_{S} F_{S T}$ \\
\hline \multirow{2}{*}{ NM_022336 } & \multirow{2}{*}{ EDAR } & \multirow{2}{*}{604095} & 2 & 108969448 & 109064345 & 0.9849 & 0.7848 & 0.1878 & 0.0010 & 0.5289 & 0.5460 & 0.9915 \\
\hline & & & \multicolumn{10}{|c|}{ ectodysplasin A receptor } \\
\hline
\end{tabular}

\begin{tabular}{|c|c|c|c|c|c|c|c|c|c|c|}
\hline \multicolumn{11}{|c|}{ SNP statistics } \\
\hline$r s \# d b S N P$ & Chr & Position & Location & HET (A) & HET (c) & $\operatorname{HET}(Y)$ & iHS (A) & iHs (c) & $\mathrm{iHS}(\mathrm{Y})$ & $F_{S T}$ \\
\hline rs10865025 dbSNP & 2 & 108970283 & Gene_3' UTR & 0.0849 & 0.2432 & 0.4265 & -2.9684 & -0.9835 & -1.5799 & 0.6008 \\
\hline rs10206737 dbSNP & 2 & 108970882 & Gene_3' UTR & 0.0000 & 0.0000 & 0.1388 & - & - & -0.4985 & 0.1222 \\
\hline rs3749096 dbSNP & 2 & 108970946 & Gene_3' UTR & 0.0000 & 0.0644 & 0.4800 & 2.2458 & - & 1.0588 & 0.4304 \\
\hline rs3749097 dbSNP & 2 & 108971124 & Gene_3' UTR & 0.0000 & 0.0644 & 0.3299 & 2.2458 & - & 0.4297 & 0.1557 \\
\hline rs $7607563 \mathrm{dbSNP}$ & 2 & 108971839 & Gene_3' UTR & 0.0000 & 0.1932 & 0.3832 & - & 0.9484 & -0.0384 & 0.2887 \\
\hline$r s 3827760 \mathrm{dbSNP}$ & 2 & 108972119 & Gene_cDS & 0.0849 & 0.0000 & 0.0000 & -3.1570 & - & - & 0.9236 \\
\hline rs6542783 dbSNP & 2 & 108972623 & Gene & 0.0000 & 0.0644 & 0.4800 & 2.4794 & - & 1.0233 & 0.4304 \\
\hline
\end{tabular}

Figure 2

Using EDAR to demonstrate the output of a data query. After submitting EDAR as the search term, all hit data are provided in three tables. The regional statistics indicate whether the query is localized in a low HET region in a certain population or in a highly differentiated region among populations. The gene table with the sequence accession ID, gene symbol, and OMIM ID provides $E_{S}$ values of HET and $P$-value of iHS of each HapMap geographical group, as well as the $E_{S}$ value of $F_{S T}$ to show population differentiation. Finally, in addition to the rs\# and its locations at both the chromosome and gene, SNPs of EDAR are displayed with individual HET and iHS of each population followed with $F_{S T}$. The number above the third table indicates the total number of the SNPs identified in the query (only the first 7 were shown in the figure in this example). To illustrate selection related signals, the SNP outliers of iHS and $F_{S T}$, as well as the extra $E_{S}$ values $(>0.95)$ of $H E T$ and $F_{S T}$, are highlighted in the tables.

loci, the regional HET is linear to Tajima's D [see Additional file 1]; therefore, we present HET data only in SNP@Evolution. Considering that iHS was derived from $\mathrm{EHH}$ and $\mathrm{iHH}$, we have chosen iHS to be the measurement of haplotype diversity in our database.

\section{Utility and Discussion}

There are two user interfaces in SNP@Evolution, one for data query and another for data visualization. In the data query interface, users submit one or more SNP rs\# labels, gene symbols, gene sequence accession IDs, or specific chromosome regions. Results are displayed in three tables, as shown in the EDAR example in Fig. 2. Measurements--including the highly differentiated region among three geographical groups, $\mathrm{E}_{\mathrm{S}}$ values of $\mathrm{HET}, F_{S T}$, and $P$ value of iHS of the gene, as well as the HET, $F_{S T}$, and iHS of the SNPs--indicate that the EDAR gene is under positive selection in Asians as previously reported [19,31]. Like all queries, these EDAR results can be downloaded in a table formatted file.

Table I: The distribution of $F_{S T}$ outliers across autosomes in data of HapMap Phase II Build36

\begin{tabular}{|c|c|c|c|c|c|c|c|c|}
\hline & & \multicolumn{5}{|c|}{ Gene } & \multirow[b]{2}{*}{ Intergenic region } & \multirow[b]{2}{*}{ Total } \\
\hline & & 5' UTR & CDS* & Intron & 3' UTR & Total & & \\
\hline \multirow{2}{*}{ SNP } & Outlier & 59 & 420 & 12,598 & 299 & 13,370 & 20,225 & 33,601 \\
\hline & All & 4,604 & 43,858 & $1,280,810$ & 30,153 & $1,358,805$ & $2,259,801$ & $3,619,226$ \\
\hline Ratio (outlier/all) & & 0.0128 & 0.0096 & 0.0098 & 0.0099 & 0.0098 & 0.0089 & 0.0093 \\
\hline
\end{tabular}

* CDS, coding sequence. 


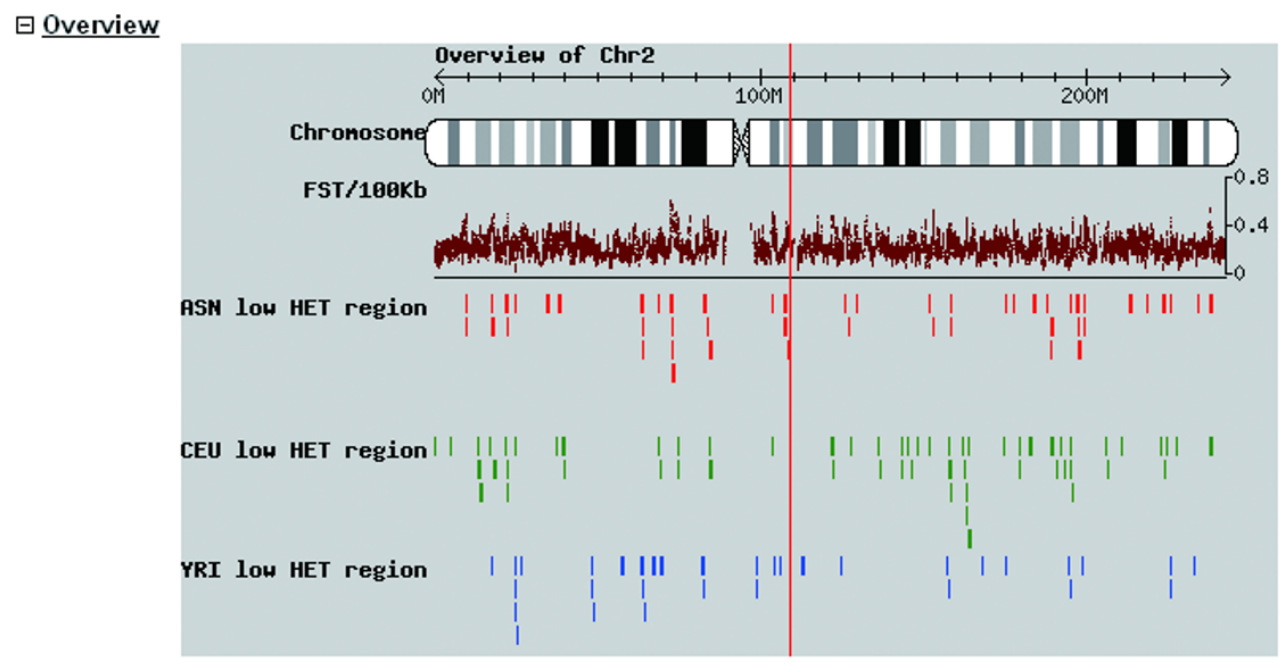

$\boxminus \underline{\text { Region }}$

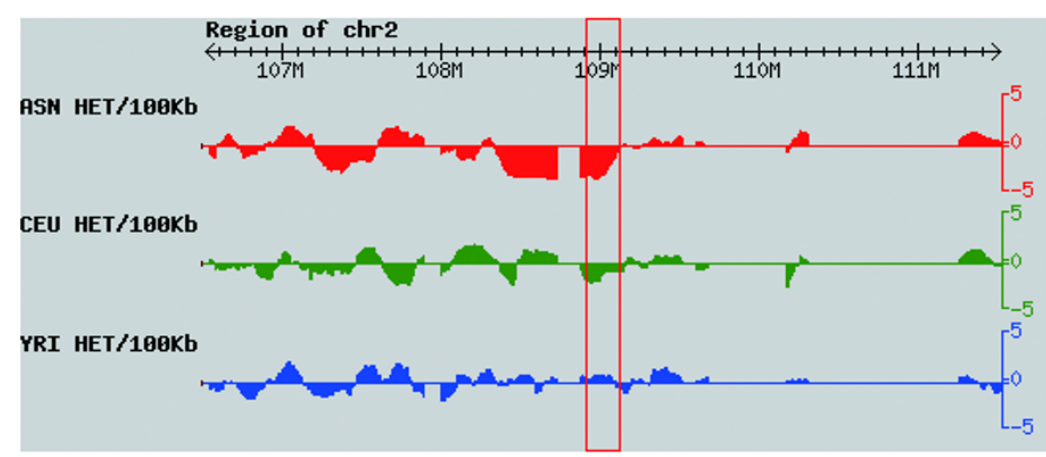

$\boxminus \underline{\text { Details }}$
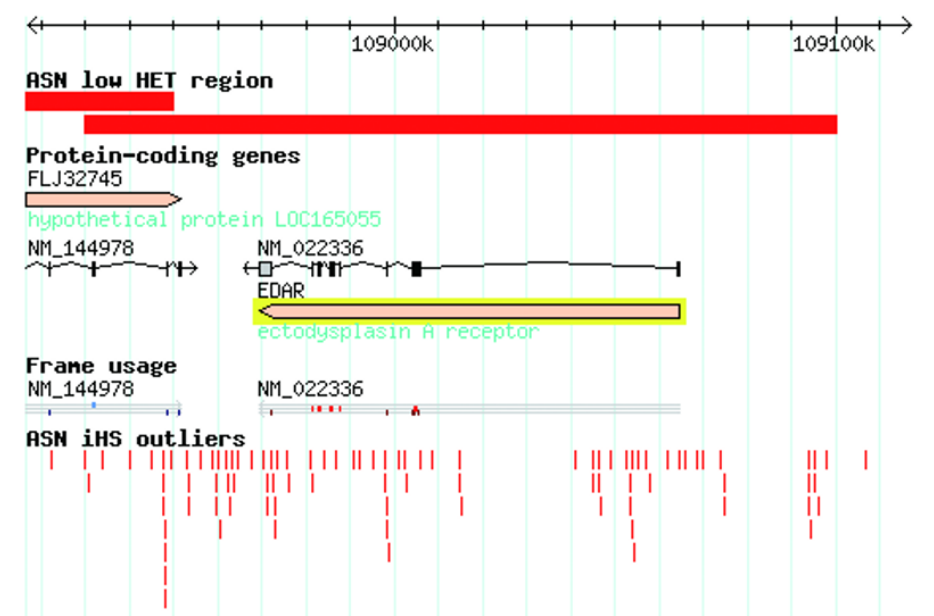

CEU iHS outliers

YRI iHS outliers

Figure 3 (see legend on next page) 
Figure 3 (see previous page)

Results of EDAR in the visualization interface. Data are illustrated in three parts as Overview (chromosome scale), Region (area of searching target and flanking sequences), and Details (searching target). The Overview shows the $F_{S T}$ values of $100 \mathrm{~kb}$ sliding windows (maroon) and the low HET regions of merged window outliers in HapMap geographical group of ASN (red), CEU (green), and YRI (blue) along the ideogram of the chromosome. Region image displays the normalized HET values of $100 \mathrm{~kb}$ sliding windows that flanking the searching target in each population with the same color as illustrated in Overview. The basic line (zero) represents the average values of Normalized HET, therefore the comparatively high HET windows are shown above zero and low HET windows appear below the basic line. For the Details of the searching target, it provides (from the top to the bottom) the fine position in the chromosome, the low HET region (merged outliers of sliding windows) in the geographical group (red bar for ASN in EDAR gene), the genes (incarnadine) with the arrow pointing to the transcription direction, the transcripts and the frame usage of the genes (grey), the SNP outliers of iHS in each population as colored sticks, and at last the SNP outliers of $F_{S T}$ (maroon) to demonstrate if differentiation signals exist among three populations. For EDAR gene with a yellow label as the target gene, it is localized in a highly differentiated region among the geographical group and much more iHS outliers are in ASN than that in CEU and YRI groups. In addition, $F_{S T}$ outliers are also enriched in EDAR.

To better visualize the data, we implemented the Generic Model of Organism Database (GMOD) tool [32]. This interface uses similar input as described above. As shown in Fig. 3, each search result is shown as three parts as the Overview, the Region, and the Details. First, along the electronic G-stain of a given chromosome, $F_{S T}$ values of $100 \mathrm{~kb}$ sliding windows are depicted as scatter plots, and the low HET regions which were merged from adjacent window outliers are plotted as block diagrams (Fig. 3, top). Normalized HET values of $100 \mathrm{~kb}$ sliding windows are enlarged and illustrated as histograms (Fig. 3, middle). These figures allow one to directly observe the polymorphism and population differentiation patterns in chromosomes. In order to help locate positive selection signals throughout the genome, the SNP outliers of $F_{S T}$ and iHS are displayed as sticks in different colors along the searching regions, with additional gene annotation (Fig. 3, bottom). These features are provided with information of the three geographical groups shown in different colors.

Furthermore, results in the data query interface can be easily linked to the visualization interface to see the features under the background of the chromosome. To help users obtain additional information, we also provide the SNP links to HapMap database and NCBI dbSNP database and the gene links to Entrez Nucleotide and OMIM. To facilitate the operation, a page of 'Complete Guide' is included for detailed introduction and efficient use of SNP@Evolution. The terms used in measurements and population groups in this database are hyperlinked to the 'Abbreviations' page for brief reference.

Generally speaking, SNP@Evolution allows users to access all data through the 'Download' link. In these datasets, most of computations were conducted based on HapMap and related projects. As the data came from no more than a hundred of individuals in each HapMap population group, one shall also consider the sample size while making conclusions. The aim of SNP@Evolution is to provide genome wide signals of positive selection on human being, to generate fine scale traces of natural selection in enlarged samples is a long term goal in our research and computation.

\section{Conclusion}

SNP@Evolution is a valuable and useful resource for finding and verifying signals of natural selection, and we will continue to update SNP@Evolution as research in positive selection progresses. At regions showing strong selection signals, we plan to add additional SNP information obtained from resequencing data from our work and from public datasets. Genotype data of new individuals from various sources will also be added to the database. By evaluating the effects of amino acid substitution with the method of Sorting Intolerant From Tolerant (SIFT) [33], we also plan to include protein functions at genic regions showing strong selection signals.

\section{Availability and requirements}

SNP@Evolution is freely available at http://bighap map.big.ac.cn/. A minimum screen resolution of 1,152 × 864 is recommended. Please send all questions, comments, and suggestions to chengf@big.ac.cn.

\section{Authors' contributions}

The original data process and computation, webpage design, and manuscript preparation were undertaken by FC. WC made great contributions to the data integration, web organization and preparation of the manuscript. ER revised the manuscript and made great improvements regarding SNP@Evolution. LD analyzed the data and made important suggestions to the structure of the database. $\mathrm{CZ}$ directed the project and prepared the bulk of the manuscript. All the authors read and approved the final manuscript. 


\section{Additional material}

\section{Additional file 1}

Linear relationship between regional HET and Tajima's D. The figure provided represents the statistical relationship between regional averaged HET and Tajima's D. The example is taken from Chromosome 22 of HapMap YRI group.

Click here for file

[http://www.biomedcentral.com/content/supplementary/14712148-9-221-S1.doc]

\section{Acknowledgements}

We thank Guanghua Wu for editing this manuscript. We also appreciate the help from Dake Zhang and Yubin Sun on website construction. This work was supported by a grant of National Natural Science Foundation of China (3089003I) to CZ.

\section{References}

I. Verrelli BC, McDonald JH, Argyropoulos G, Destro-Bisol G, Froment A, Drousiotou A, Lefranc G, Helal AN, Loiselet J, Tishkoff SA: Evidence for balancing selection from nucleotide sequence analyses of human G6PD. American journal of human genetics 2002, 7I(5): IIII2-II28

2. Bersaglieri T, Sabeti PC, Patterson N, Vanderploeg T, Schaffner SF, Drake JA, Rhodes M, Reich DE, Hirschhorn JN: Genetic signatures of strong recent positive selection at the lactase gene. American journal of human genetics 2004, 74(6): I I I I- I I 20

3. Mekel-Bobrov N, Gilbert SL, Evans PD, Vallender EJ, Anderson JR, Hudson RR, Tishkoff SA, Lahn BT: Ongoing adaptive evolution of ASPM, a brain size determinant in Homo sapiens. Science. 2005, 309(574I): I720-1722.

4. Biswas S, Akey JM: Genomic insights into positive selection. Trends Genet 2006, 22(8):437-446.

5. Ronald J, Akey JM: Genome-wide scans for loci under selection in humans. Human genomics 2005, 2(2): I I 3-125.

6. Wang ET, Kodama G, Baldi P, Moyzis RK: Global landscape of recent inferred Darwinian selection for Homo sapiens. Proceedings of the National Academy of Sciences of the United States of America 2006, I03(I): |35-| 40.

7. The International HapMap Consortium: A haplotype map of the human genome. Nature 2005, 437(7063): I 299-| 320.

8. Frazer KA, Ballinger DG, Cox DR, Hinds DA, Stuve LL, Gibbs RA Belmont JW, Boudreau A, Hardenbol P, Leal SM, et al:: A second generation human haplotype map of over 3.I million SNPs. Nature 2007, 449(7 I 64):85I-86I

9. Conrad DF, Andrews TD, Carter NP, Hurles ME, Pritchard JK: A high-resolution survey of deletion polymorphism in the human genome. Nature genetics 2006, 38(I):75-8I.

10. lafrate AJ, Feuk L, Rivera MN, Listewnik ML, Donahoe PK, Qi Y, Scherer SW, Lee C: Detection of large-scale variation in the human genome. Nature genetics 2004, 36(9):949-95I.

II. Birney E, Stamatoyannopoulos JA, Dutta A, Guigo R, Gingeras TR, Margulies EH, Weng Z, Snyder M, Dermitzakis ET, Thurman RE, et al.: Identification and analysis of functional elements in $1 \%$ of the human genome by the ENCODE pilot project. Nature 2007, 447(7 | 46):799-8I6

12. Rakyan VK, Hildmann T, Novik KL, Lewin J, Tost J, Cox AV, Andrews TD, Howe KL, Otto T, Olek A, et al: DNA methylation profiling of the human major histocompatibility complex: a pilot study for the human epigenome project. PLOS Biol 2004, 2(I 2):e405.

13. Redon R, Ishikawa S, Fitch KR, Feuk L, Perry GH, Andrews TD, Fiegler $\mathrm{H}$, Shapero $\mathrm{MH}$, Carson AR, Chen W, et al.: Global variation in copy number in the human genome. Nature 2006, 444(7 I I 8): 444-454.

14. Stranger BE, Forrest MS, Dunning M, Ingle CE, Beazley C, Thorne N, Redon R, Bird CP, de Grassi A, Lee C, et al.: Relative impact of nucleotide and copy number variation on gene expression phenotypes. Science. 2007, 3 I 5(58 I 3):848-853.

15. Stranger BE, Forrest MS, Clark AG, Minichiello MJ, Deutsch S, Lyle R, Hunt S, Kahl B, Antonarakis SE, Tavare S, et al:: Genome-wide associations of gene expression variation in humans. PLoS genetics 2005, I(6):e78.

16. Dixon AL, Liang L, Moffatt MF, Chen W, Heath S, Wong KC, Taylor J, Burnett E, Gut I, Farrall M, et al:: A genome-wide association study of global gene expression. Nature genetics 2007 , 39(I0): I202-I 207

17. McCarroll SA, Hadnott TN, Perry GH, Sabeti PC, Zody MC, Barrett JC, Dallaire S, Gabriel SB, Lee C, Daly MJ, et al.: Common deletion polymorphisms in the human genome. Nature genetics 2006 , 38(I):86-92.

18. Akey JM, Zhang G, Zhang K, Jin L, Shriver MD: Interrogating a high-density SNP map for signatures of natural selection. Genome research 2002, I 2(12):1805-1814.

19. Sabeti PC, Varilly P, Fry B, Lohmueller J, Hostetter E, Cotsapas C, Xie X, Byrne EH, McCarroll SA, Gaudet R, et al.: Genome-wide detection and characterization of positive selection in human populations. Nature 2007, 449(7 I 64):913-918.

20. Shriver MD, Kennedy GC, Parra EJ, Lawson HA, Sonpar V, Huang J, Akey JM, Jones KW: The genomic distribution of population substructure in four populations using $\mathbf{8 , 5 2 5}$ autosomal SNPs. Human genomics 2004, I(4):274-286.

21. Shriver MD, Mei R, Parra El, Sonpar V, Halder I, Tishkoff SA, Schurr TG, Zhadanov SI, Osipova LP, Brutsaert TD, et al.: Large-scale SNP analysis reveals clustered and continuous patterns of human genetic variation. Human genomics 2005, 2(2):81-89.

22. Carlson CS, Thomas DJ, Eberle MA, Swanson JE, Livingston RJ, Rieder M], Nickerson DA: Genomic regions exhibiting positive selection identified from dense genotype data. Genome research 2005, I 5( I I): I553-I565.

23. Weir BS, Cardon LR, Anderson AD, Nielsen DM, Hill WG: Measures of human population structure show heterogeneity among genomic regions. Genome research 2005 , I5(I I): |468-|476.

24. Sabeti PC, Reich DE, Higgins JM, Levine HZ, Richter DJ, Schaffner SF, Gabriel SB, Platko JV, Patterson NJ, McDonald G], et al.: Detecting recent positive selection in the human genome from haplotype structure. Nature 2002, 419(6909):832-837.

25. Voight BF, Kudaravalli S, Wen X, Pritchard JK: A map of recent positive selection in the human genome. PLoS Biol 2006, 4(3):e72.

26. Park J, Hwang S, Lee YS, Kim SC, Lee D: SNP@Ethnos: a database of ethnically variant single-nucleotide polymorphisms. Nucleic acids research 2007:D7II-7I5.

27. Spencer CC, Coop G: SelSim: a program to simulate population genetic data with natural selection and recombination. Bioinformatics 2004, 20( I 8):3673-3675.

28. Kelley JL, Madeoy J, Calhoun JC, Swanson W, Akey JM: Genomic signatures of positive selection in humans and the limits of outlier approaches. Genome Res 2006, I 6(8):980-989.

29. Akey JM: Constructing genomic maps of positive selection in humans: where do we go from here? Genome Res 2009, 19(5):7| I-722.

30. Pickrell JK, Coop G, Novembre J, Kudaravalli S, Li JZ, Absher D, Srinivasan BS, Barsh GS, Myers RM, Feldman MW, et al.: Signals of recent positive selection in a worldwide sample of human populations. Genome Res 2009, I 9(5):826-837.

31. Fujimoto A, Kimura R, Ohashi J, Omi K, Yuliwulandari R, Batubara L, Mustofa MS, Samakkarn U, Settheetham-Ishida W, Ishida T, et al.: A scan for genetic determinants of human hair morphology: EDAR is associated with Asian hair thickness. Hum Mol Genet 2008, I 7(6):835-843

32. Stein LD, Mungall C, Shu S, Caudy M, Mangone M, Day A, Nickerson E, Stajich JE, Harris TW, Arva A, et al:: The generic genome browser: a building block for a model organism system database. Genome research 2002, I 2(10):1599-1610.

33. Ng PC, Henikoff S: SIFT: Predicting amino acid changes that affect protein function. Nucleic acids research 2003, 3 I (13):38|2-38|4 